\title{
The FasT-Fix Repair Technique for Ramp Lesion of the Medial Meniscus
}

\author{
Wei-ping Li, Zhong Chen, Bin Song, Rui Yang, MD, and Weiquan Tan
}

Department of Sport Medicine Center, Sun Yat-Sen Memorial Hospital, Guangzhou, China

\begin{abstract}
Introduction: This technical note describes a new arthroscopic technique to repair the peripheral attachment lesion of the posterior horn of the medial meniscus. The operation was performed under arthroscopy using a standard anterior portal.

Surgical Technique: A FasT-Fix needle was inserted obliquely close to the tibial plateau and the first implant was inserted into the joint capsule depending on its bending angle underneath the meniscus. The second implant was inserted through $1 / 3$ periphery of the meniscus into the meniscocapsular area. The pre-tied self-sliding knot was tensioned to achieve secure fixation of the posterior meniscal peripheral attachment at the original attachment point.

Materials and Methods: From August 2011 to February 2014, 23 knees were diagnosed as ramp lesion, underwent meniscal repair using FasT-Fix technique.

Results: All patients were followed up for average 14 months. The Lysholm score improved from preoperative $64.4 \pm 4.52$ to postoperative $91.2 \pm 4.60$. Conclusions: We believe that the FasT-Fix technique via the standard anterior portal can be a more convenient and less traumatic alternative for repair of the peripheral attachment lesion of the posterior horn of the medial meniscus in the anterior cruciate ligament deficient knee.
\end{abstract}

Keywords: Knee, Anterior cruciate ligament, Medial meniscus, Ramp lesion

\section{Introduction}

The peripheral attachment lesion of the posterior horn of the medial meniscus is termed as "ramp lesion". Usually, it is insidiously displayed as meniscocapsular avulsion, meniscotibial ligament avulsion, or combination of the two types of injuries. It frequently occurs after an anterior cruciate ligament (ACL) tear ${ }^{1)}$ with an incidence of $9.3 \%-17 \%^{1,2)}$. If neglected or misdiagnosed, a ramp lesion would bring about instability or injury of the body of the medial meniscus. Then, the "wedged" resistance of the medial meniscus would be withdrawn, resulting in anteroposte-

Received August 29, 2014; Revised October 26, 2014;

Accepted November 20, 2014

Correspondence to: Zhong Chen, MD

Department of Sport Medicine Center, Sun Yat-Sen Memorial Hospital,

107 Yan Jiang West Road, Guangzhou 510-120, China

Tel: +86-186-0577-7992

E-mail: z62587253@163.com

This is an Open Access article distributed under the terms of the Creative Commons Attribution Non-Commercial License (http://creativecommons.org/licenses/by-nc/3.0/) which permits unrestricted non-commercial use, distribution, and reproduction in any medium, provided the original work is properly cited. rior instability or anteromedial rotatory laxity of the knee $e^{1,3,4)}$ and even ACL reconstruction failure. Suture hook through a high posteromedial portal is a commonly used meniscal technique for a ramp lesion ${ }^{1,3,5-8)}$. There have been no studies on this special lesion repaired using the arthroscopic FasT-Fix (Smith \& Nephew Endoscopy, Andover, MA, USA) technique under the view from the posteromedial compartment deeply into the notch via the anterolateral portal only.

\section{Surgical Technique}

\section{Surgical Exploration (Schematic Diagram A)}

During procedure, the patients were placed supine on the operating table with a tourniquet placed high on the thigh. The knee was placed at $90^{\circ}$ of flexion with a foot support to allow for full range of knee motion. We used a standard anterolateral portal for arthroscopic visualization of the medial compartment and posteromedial compartment in which a ramp lesion was located, and an anteromedial portal for the instruments.

1) Step 1: the view of the medial compartment

To gain access to the medial compartment, the presence of a www.jksrr.org 
meniscal substance tear was evaluated via the anteromedial portal with meticulous probing of the meniscal tissue. The ramp lesion could be assessed preliminarily and the probe was kept resting in the field of posterior horn of the medial meniscus if abnormality was found (Fig. 1A).

\section{2) Step 2: the view of the intercondylar notch}

To gain access to the posteromedial compartment, the arthroscope was introduced through the anterolateral portal and advanced into the posteromedial compartment through the intercondylar notch and underneath the posterior cruciate ligament (PCL). Arthroscopy was performed with a $2.7-\mathrm{mm}$ diameter $70^{\circ}$ arthroscope, if necessary, to protect the PCL and to obtain a wider view of the posteromedial compartment for identification of the ramp lesion close to the posterior $1 / 3$ of the meniscus. Under arthroscopic view, a probe was used to explore the peripheral attachment of the posterior horn of the medial meniscus through the anterolateral portal and to confirm the type of the ramp lesion (Fig. 1B).

\section{Surgical Technique (Schematic Diagrams B and C)}

To ensure optimal visualization for repair of the ramp lesion, it was needed to switch arthroscopic view from Step 1 to Step 2, or reversely, to avoid a chondral injury during instrumentation. But, the anterolateral portal for the arthroscope and the anteromedial portal for the instruments were still unchanged. During instrumentation, the torn peripheral attachment of the posterior horn of the medial meniscus was vascularized to produce vascular surface to stimulate the healing response by rasp (Fig. 1B), and a split cannula was used to facilitate safe insertion of the FasT-Fix device into the knee, which was advanced to the field of ramp lesion under visualization of the medial compartment. The ramp lesion
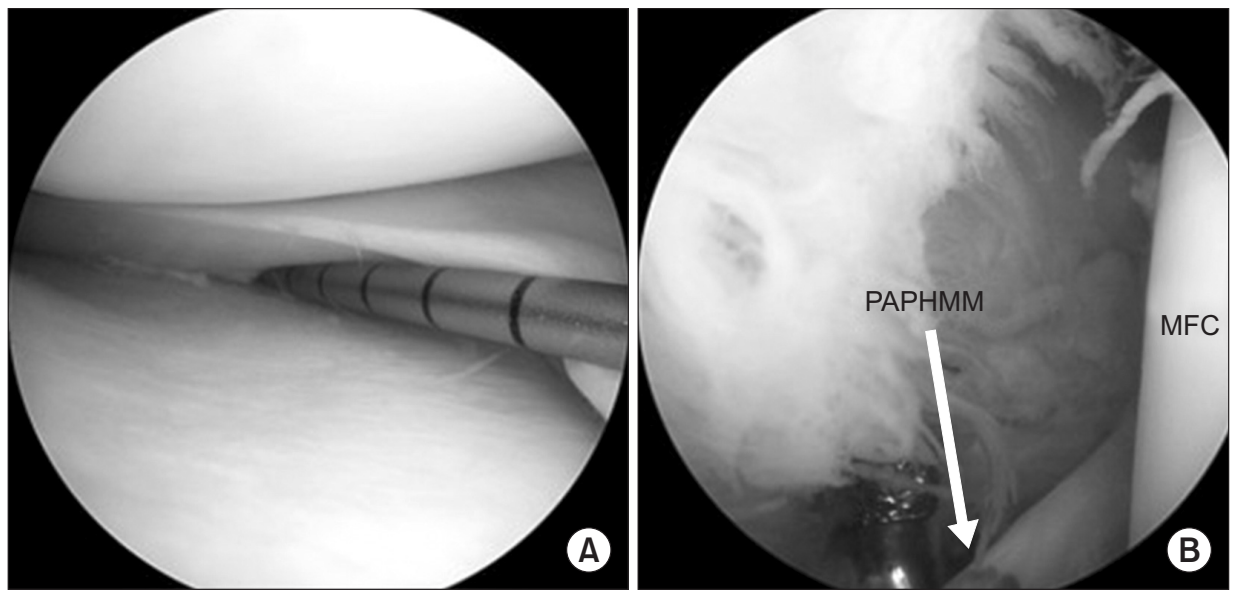

A

Step 2: view of intercondylar notch

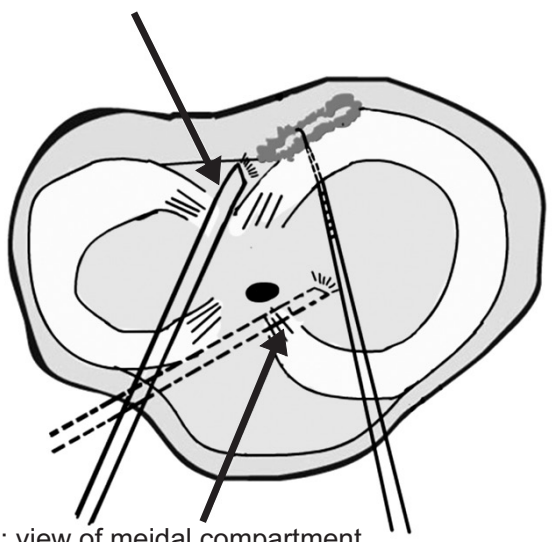

B

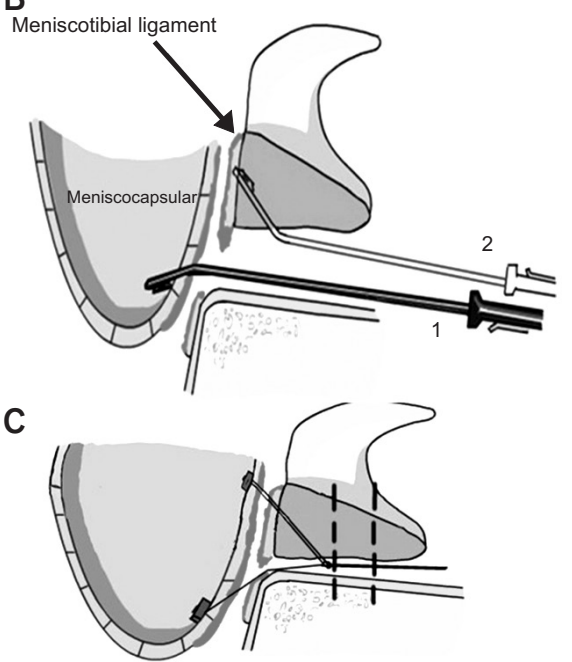

Fig. 1. (A) Confirming the peripheral attachment lesion of the posterior horn of the medial meniscus using a probe via the view of medial compartment. (B) The meniscocapsular tear and meniscotibial ligament tear were concomitantly explored via the view of posteromedial compartment (The peripheral attachment cleft of the posterior horn of the medial meniscus was vascularized by rasp). PAPHMM: peripheral attachment of the posterior horn of the medialmeniscus, MFC: medial femoral condyle.

Fig. 2. (A) Schematic cross section view shows meniscocapsular \& meniscotibial ligament tear repaired by FasT-Fix device. (B) Schematic sagittal view shows that the needle was used to obliquely insert the first implant into joint capsule underneath meniscus and the second implant inserted via $1 / 3$ periphery meniscus was fixed on the joint capsule. (C) Schematic sagittal view shows that the pre-tied self-sliding knot was gradually tensioned, achieving secure fixation of the posterior meniscal peripheral attachment at the original attachment. 
was fixed by the FasT-Fix device (Fig. 2B) via the anteromedial portal under alternate visualization of the medial compartment and the intercondylar notch. To bring the capsular synovium as far as possible to the attachment site, a leg holder was used to flex or extend the knee with valgus force. The surgeon repaired the ramp lesion until the capsular synovium was seen proximal to the original place ${ }^{3,7}$. Each FasT-Fix depth limiter was trimmed, the real and valid part of the needle was left, and the curve was increased manually. The curve allows for better approach below the meniscus while avoiding damage to the femoral condyle. The needle close to the tibial plateau was used to obliquely insert the first implant into the joint capsule depending on its curve underneath the meniscus and the second implant inserted via the $1 / 3$ periphery meniscus was fixed on the capsule (Figs. 2 and 3). The pre-tied self-sliding knot was tensioned to achieve secure fixation of the posterior meniscal peripheral attachment at the original attachment (Fig. 4). Similar to repair of a meniscal substance tear, 3-5 $\mathrm{mm}$ was recommended as the interval of suture materials.

\section{Materials and Methods}

From August 2011 to February 2014, 23 knees were diagnosed as ramp lesion and ACL injury simultaneously, underwent meniscal repair using FasT-Fix technique with concomitant ACL reconstruction. All patients started non-weight-bearing motion immediately postoperatively and full weight-bearing was permitted at 6 weeks postoperatively. For patients in whom edema disappeared, a hinged brace was applied and non-weight-bearing motion was restricted to $0^{\circ}-90^{\circ}$ at 4 weeks postoperatively. Full weight-bearing and full range of motion was permitted at 6 weeks postoperatively. Jogging was permitted at 6 months postoperatively. All patients were evaluated Lysholm score postoperatively.

\section{Results}

All patients were followed up for 6 to 27 months (average 14 months). The Lysholm score improved from preoperative $64.4 \pm 4.52$ to postoperative $91.2 \pm 4.60$, and the difference was sta-
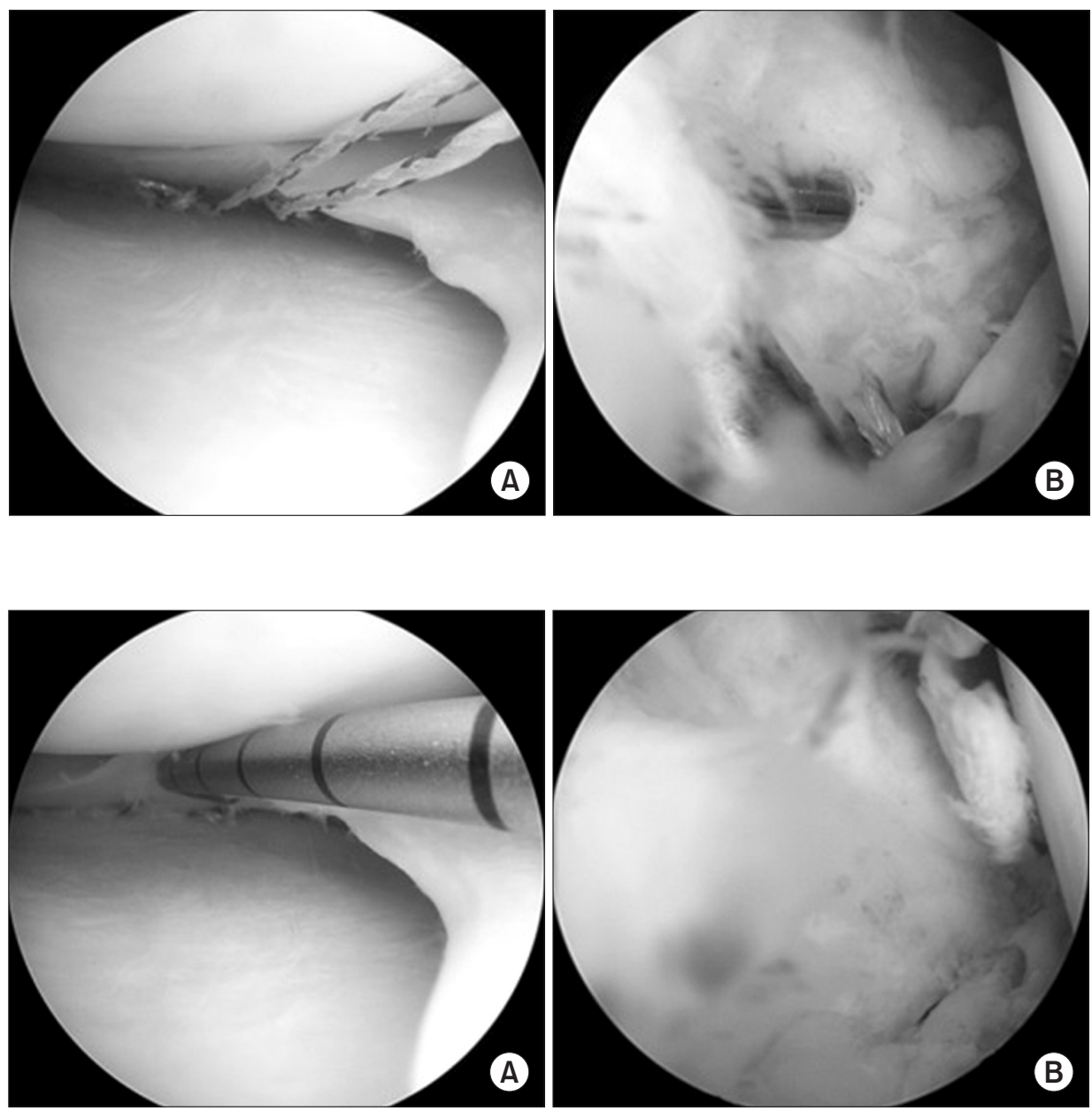

Fig. 3. (A) The meniscus was fixed by two FasT-Fix devices under inferior surface. (B) Two implants were inserted into the meniscocapsular area (before pre-tied selfsliding knot was tensioned).
Fig. 4. (A) Two pre-tied self-sliding knots were tensioned and the meniscal cleft was closed (view of medial compartment). (B) The meniscocapsular area was tensioned and a peripheral attachment lesion of the posterior horn of the medial meniscus was repaired by arthroscopic FasT-Fix technique (view of posteromedial compartment). 
tistically significant $(\mathrm{p}<0.05)$. The clinical assessment was similar to suture hook technique which reported by other authors ${ }^{1,6,7)}$.

\section{Discussion}

Ramp lesions commonly result from an acute ACL rupture or chronic ACL deficiency according to several retrospective clinical studies ${ }^{1,25)}$ and biomechanical studies ${ }^{9)}$. For the abovementioned reasons, the meniscus laxity or tear could be a ramp lesion occurring either at the moment of initial injury or following the progressive stretching of ACL deficiency, since then the "wedged" resistance of medial meniscus would be withdrawn, resulting in anteroposterior instability, anteromedial rotatory laxity of the knee $^{1,3,4)}$, or even ACL reconstruction failure.

Repair of the $1 / 3$ peripheral meniscus in conjunction with ACL reconstruction has been reported to produce favorable meniscal healing ${ }^{10)}$; therefore, the ramp lesion area located at the peripheral attachment of the meniscus has high healing capacity.

Conventional all-inside suture hook arthroscopic technique was widely used for ramp lesion repair ${ }^{6,7)}$. However, we consider that this technique cannot precisely restore sloped capsular synovium to the original attachment point anatomically. Furthermore, it necessitates an additional posteromedial portal for exploration and operation carries the risk of iatrogenic trauma. Most importantly, suture hook technique is difficult to perform for junior surgeons and requires prolonged operation time. Therefore, development of new techniques for the repair of ramp lesions is necessary.

The new all-inside suture arthroscopic technique, FasT-Fix technique, uses a tiny implant for suturing peripheral attachment of the posterior horn of the medial meniscus via the standard anterior portal only because it is more convenient for the surgeon. Ramp lesions were directly repaired using "0" non-absorbable polyester wire and polymer anchor without rejection of the suture material. This alternative method obviously reduces the risk of neurovascular complications but is time-consuming.

Finally, we realized that the study had some considerable limitations. First, it was possible to miss the hidden ramp lesion ${ }^{5)}$ because we only used the anterolateral portal for exploration. Thus, we did conventionally fixate the inferior meniscus to the meniscocapsular area when meniscocapsular laxity or pure meniscotibial ligament avulsion was found, but we did not agree with Sonnery-Cottet et al..$^{5)}$ who advised minimal debridement of the superficial meniscocapsular area. Second, FasT-Fix device carries the risk ${ }^{11)}$ of implant breakage, synovitis, or migration of the implant. Third, an injury to the cartilage of the medial tibial plateau may be a possible complication ${ }^{12}$. The repair technique should be improved, and clinical and second-look arthroscopic evaluation should be included in further studies.

\section{Conclusions}

In conclusion, we present a new technique for all-inside arthroscopic repair of the peripheral attachment of the posterior horn of the medial meniscus in order to facilitate operation, prevent iatrogenic trauma, and retain the functional integrity of the meniscus, while avoiding progression into meniscal laxity or tear and influence on ACL reconstruction.

\section{Conflict of Interest}

No potential conflict of interest relevant to this article was reported.

\section{References}

1. Liu X, Feng H, Zhang H, Hong L, Wang XS, Zhang J. Arthroscopic prevalence of ramp lesion in 868 patients with anterior cruciate ligament injury. Am J Sports Med. 2011;39: 832-7.

2. Bollen SR. Posteromedial meniscocapsular injury associated with rupture of the anterior cruciate ligament: a previously unrecognised association. J Bone Joint Surg Br. 2010;92:222-3.

3. Mariani PP. Posterior horn instability of the medial meniscus a sign of posterior meniscotibial ligament insufficiency. Knee Surg Sports Traumatol Arthrosc. 2011;19:1148-53.

4. Ahn JH, Bae TS, Kang KS, Kang SY, Lee SH. Longitudinal tear of the medial meniscus posterior horn in the anterior cruciate ligament-deficient knee significantly influences anterior stability. Am J Sports Med. 2011;39:2187-93.

5. Sonnery-Cottet B, Conteduca J, Thaunat M, Gunepin FX, Seil R. Hidden lesions of the posterior horn of the medial meniscus: a systematic arthroscopic exploration of the concealed portion of the knee. Am J Sports Med. 2014;42:921-6.

6. Ahn JH, Lee YS, Yoo JC, Chang MJ, Koh KH, Kim MH. Clinical and second-look arthroscopic evaluation of repaired medial meniscus in anterior cruciate ligament-reconstructed knees. Am J Sports Med. 2010;38:472-7.

7. Ahn JH, Wang JH, Yoo JC. Arthroscopic all-inside suture repair of medial meniscus lesion in anterior cruciate ligament: deficient knees: results of second-look arthroscopies in 39 cases. Arthroscopy. 2004;20:936-45.

8. Park IS, Kim SJ. New meniscus repair technique for periph- 
eral tears near the posterior tibial attachment of the posterior horn of the medial meniscus. Arthroscopy. 2006;22:908.

9. Markolf KL, Jackson SR, McAllister DR. Force measurements in the medial meniscus posterior horn attachment: effects of anterior cruciate ligament removal. Am J Sports Med. 2012;40:332-8.

10. Tachibana Y, Sakaguchi K, Goto T, Oda H, Yamazaki K, Iida S. Repair integrity evaluated by second-look arthroscopy after arthroscopic meniscal repair with the FasT-Fix during anterior cruciate ligament reconstruction. Am J Sports Med. 2010;38:965-71.

11. Barber FA, Schroeder FA, Oro FB, Beavis RC. FasT-Fix meniscal repair: mid-term results. Arthroscopy. 2008;24: 1342-8.

12. Kelly JD 4th, Ebrahimpour P. Chondral injury and synovitis after arthroscopic meniscal repair using an outside-in mulberry knot suture technique. Arthroscopy. 2004;20:e49-52. 